Network Working Group

Request for Comments: 4671

D. Nelson

Obsoletes: 2621

Enterasys Networks

Category: Informational

\title{
RADIUS Accounting Server MIB for IPv6
}

Status of This Memo

This memo provides information for the Internet community. It does not specify an Internet standard of any kind. Distribution of this memo is unlimited.

Copyright Notice

Copyright (C) The Internet Society (2006).

Abstract

This memo defines a set of extensions that instrument RADIUS accounting server functions. These extensions represent a portion of the Management Information Base (MIB) for use with network management protocols in the Internet community. Using these extensions, IP-based management stations can manage RADIUS accounting servers.

This memo obsoletes RFC 2621 by deprecating the MIB table containing IPv4-only address formats and defining a new table to add support for version-neutral IP address formats. The remaining MIB objects from RFC 2621 are carried forward into this document. This memo also adds UNITS and REFERENCE clauses to selected objects. 
Table of Contents

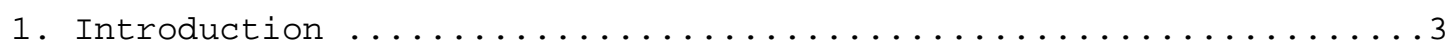

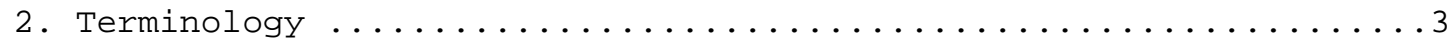

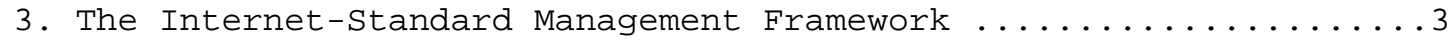

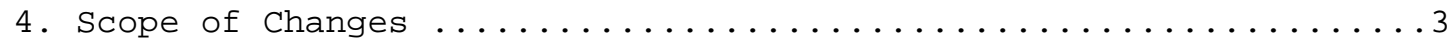

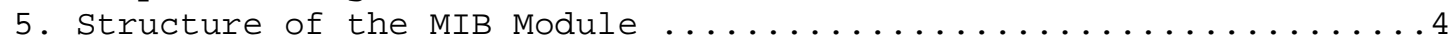

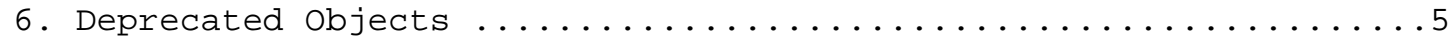

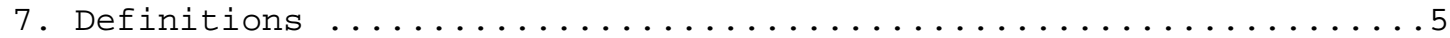

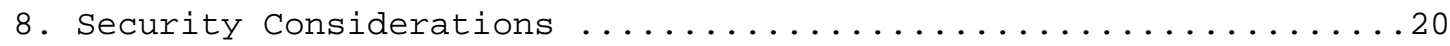

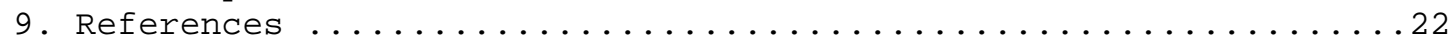

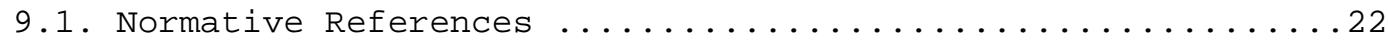

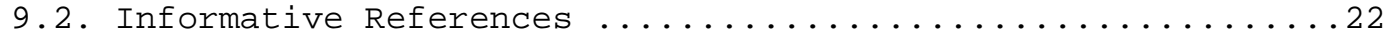

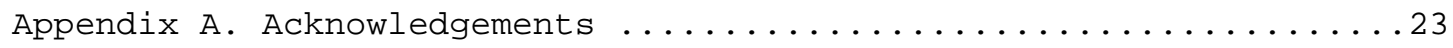




\section{Introduction}

This memo defines a portion of the Management Information Base (MIB) for use with network management protocols in the Internet community. The objects defined within this memo relate to the Remote Authentication Dial-In User Service (RADIUS) Accounting Server as defined in RFC 2866 [RFC2866].

\section{Terminology}

The key words "MUST", "MUST NOT", "REQUIRED", "SHALL", "SHALL NOT", "SHOULD", "SHOULD NOT", "RECOMMENDED", "MAY", and "OPTIONAL" in this document are to be interpreted as described in RFC 2119 [RFC2119].

This document uses terminology from RFC 2865 [RFC2865] and RFC 2866 [RFC2866].

This document uses the word "malformed" with respect to RADIUS packets, particularly in the context of counters of "malformed packets". While RFC 2866 does not provide an explicit definition of "malformed", malformed generally means that the implementation has determined the packet does not match the format defined in RFC 2866 . Those implementations are used in deployments today, and thus set the de facto definition of "malformed".

3. The Internet-Standard Management Framework

For a detailed overview of the documents that describe the current Internet-Standard Management Framework, please refer to section 7 of RFC 3410 [RFC3410].

Managed objects are accessed via a virtual information store, termed the Management Information Base or MIB. MIB objects are generally accessed through the Simple Network Management Protocol (SNMP). objects in the MIB are defined using the mechanisms defined in the Structure of Management Information (SMI). This memo specifies a MIB module that is compliant to the SMIV2, which is described in STD 58, RFC 2578 [RFC2578], STD 58, RFC 2579 [RFC2579] and STD 58, RFC 2580 [RFC2580].

\section{Scope of Changes}

This document obsoletes RFC 2621 [RFC2621], RADIUS Accounting Server MIB, by deprecating the radiusAccClientTable table and adding a new table, radiusAccClientExtTable, containing radiusAccClientInetAddressType and radiusAccClientInetAddress. The purpose of these added MIB objects is to support version-neutral IP addressing formats. The existing table containing 
radiusAccClientAddress is deprecated. The remaining MIB objects from RFC 2621 are carried forward into this document. This memo also adds UNITS and REFERENCE clauses to selected objects.

RFC 4001 [RFC4001], which defines the SMI Textual Conventions for version-neutral IP addresses, contains the following recommendation.

'In particular, when revising a MIB module that contains IPv4 specific tables, it is suggested to define new tables using the textual conventions defined in this memo [RFC4001] that support all versions of IP. The status of the new tables SHould be "current", whereas the status of the old IP version specific tables SHOULD be changed to "deprecated". The other approach, of having multiple similar tables for different IP versions, is strongly discouraged.'

5. Structure of the MIB Module

The RADIUS accounting protocol, described in RFC 2866 [RFC2866], distinguishes between the client function and the server function. In RADIUS accounting, clients send Accounting-Requests, and servers reply with Accounting-Responses. Typically, Network Access Server (NAS) devices implement the client function, and thus would be expected to implement the RADIUS accounting client MIB, while RADIUS accounting servers implement the server function, and thus would be expected to implement the RADIUS accounting server MIB.

However, it is possible for a RADIUS accounting entity to perform both client and server functions. For example, a RADIUS proxy may act as a server to one or more RADIUS accounting clients, while simultaneously acting as an accounting client to one or more accounting servers. In such situations, it is expected that RADIUS entities combining client and server functionality will support both the client and server MIBs. The server MIB is defined in this document, and the client MIB is defined in [RFC4670].

This MIB module contains thirteen scalars as well as a single table, the RADIUS Accounting Client Table, which contains one row for each RADIUS accounting client with which the server shares a secret. Each entry in the RADIUS Accounting Client Table includes twelve columns presenting a view of the activity of the RADIUS accounting server.

This MIB imports from [RFC2578], [RFC2580], [RFC3411], and [RFC4001]. 


\section{Deprecated Objects}

The deprecated table in this MIB is carried forward from RFC 2621 [RFC2621]. There are two conditions under which it MAY be desirable for managed entities to continue to support the deprecated table:

1. The managed entity only supports IPv4 address formats.

2. The managed entity supports both IPv4 and IPv6 address formats, and the deprecated table is supported for backwards compatibility with older management stations. This option SHOULD only be used when the IP addresses in the new table are in IPv4 format and can accurately be represented in both the new table and the deprecated table.

Managed entities SHOULD NOT instantiate row entries in the deprecated table, containing IPv4-only address objects, when the RADIUS accounting client address represented in such a table row is not an IPv4 address. Managed entities SHOULD NOT return inaccurate values of IP address or SNMP object access errors for IPV4-only address objects in otherwise populated tables. When row entries exist in both the deprecated IPv4-only table and the new IP-version-neutral table that describe the same RADIUS accounting client, the row indexes SHOULD be the same for the corresponding rows in each table, to facilitate correlation of these related rows by management applications.

7. Definitions

RADIUS-ACC-SERVER-MIB DEFINITIONS ::= BEGIN

IMPORTS

MODULE-IDENTITY, OBJECT-TYPE, OBJECT-IDENTITY, Counter32, Integer32, IpAddress, TimeTicks, mib-2 FROM SNMPv2-SMI SnmpAdminString FROM SNMP-FRAMEWORK-MIB InetAddressType, InetAddress FROM INET-ADDRESS-MIB MODULE-COMPLIANCE, OBJECT-GROUP FROM SNMPV2-CONF;

radiusAcCSErvMIB MODULE-IDENTITY LAST-UPDATED "200608210000Z" - - 21 August 2006 ORGANIZATION "IETF RADIUS Extensions Working Group." CONTACT-INFO

" Bernard Aboba Microsoft One Microsoft Way Redmond, WA 98052 US 


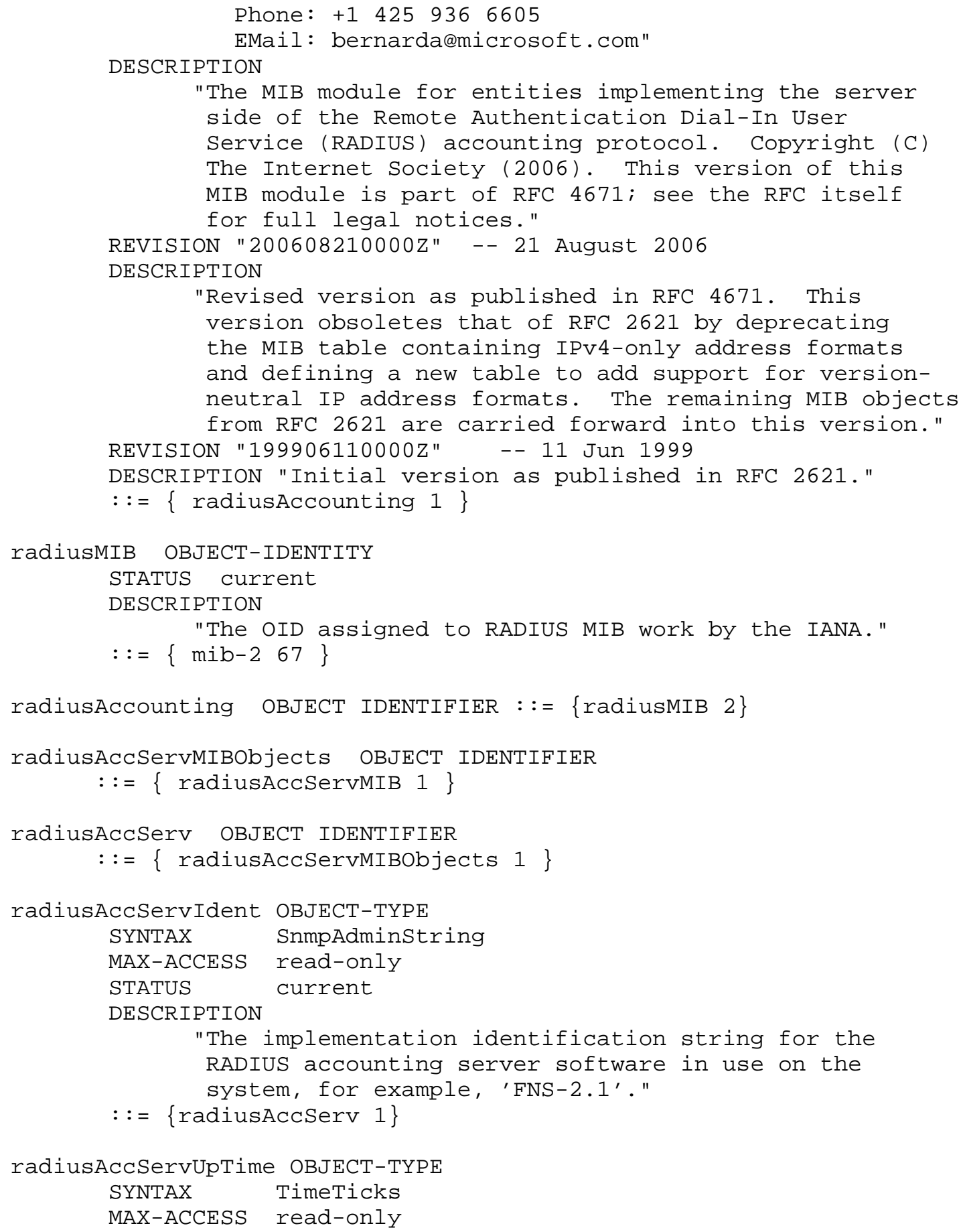

Nelson 


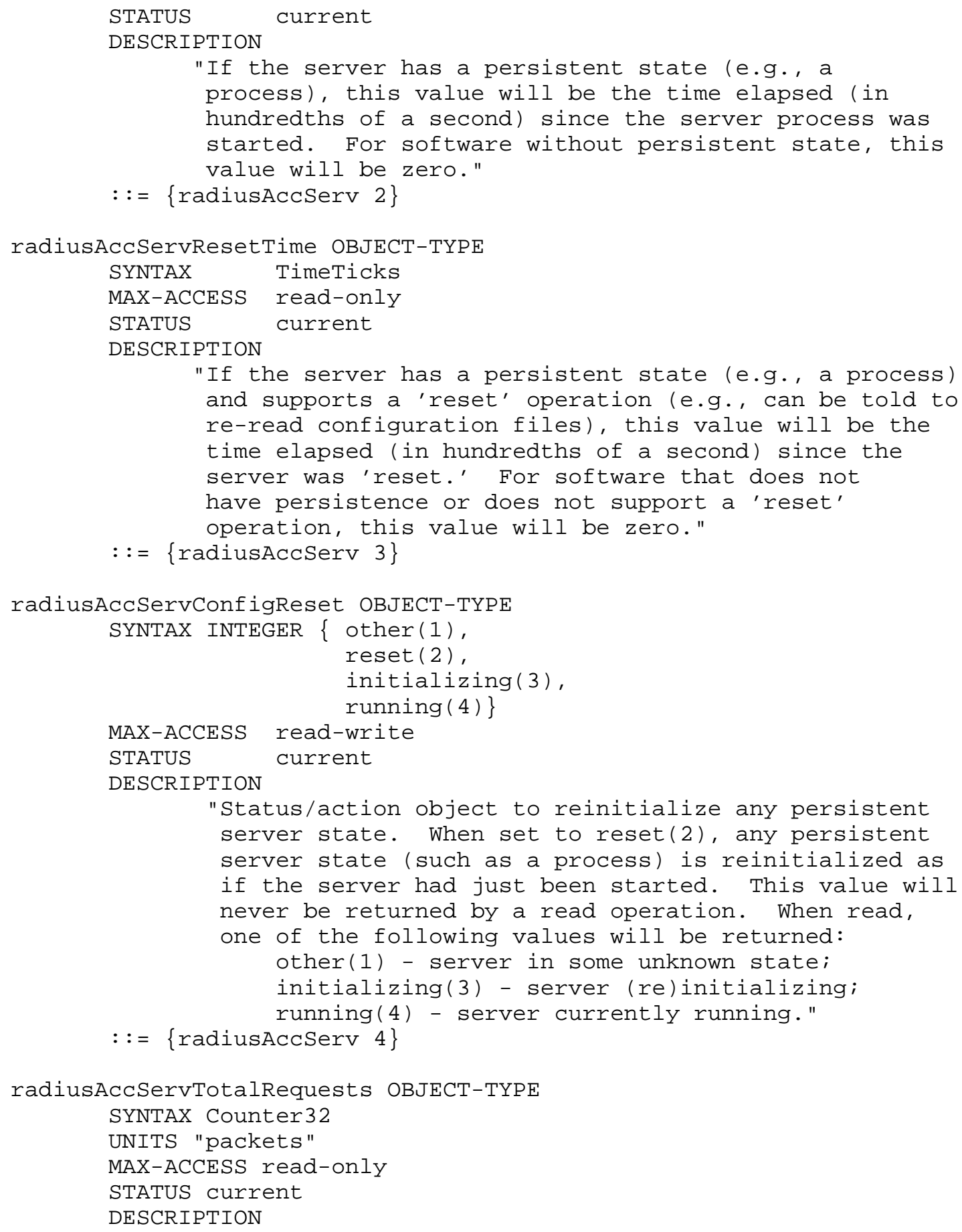




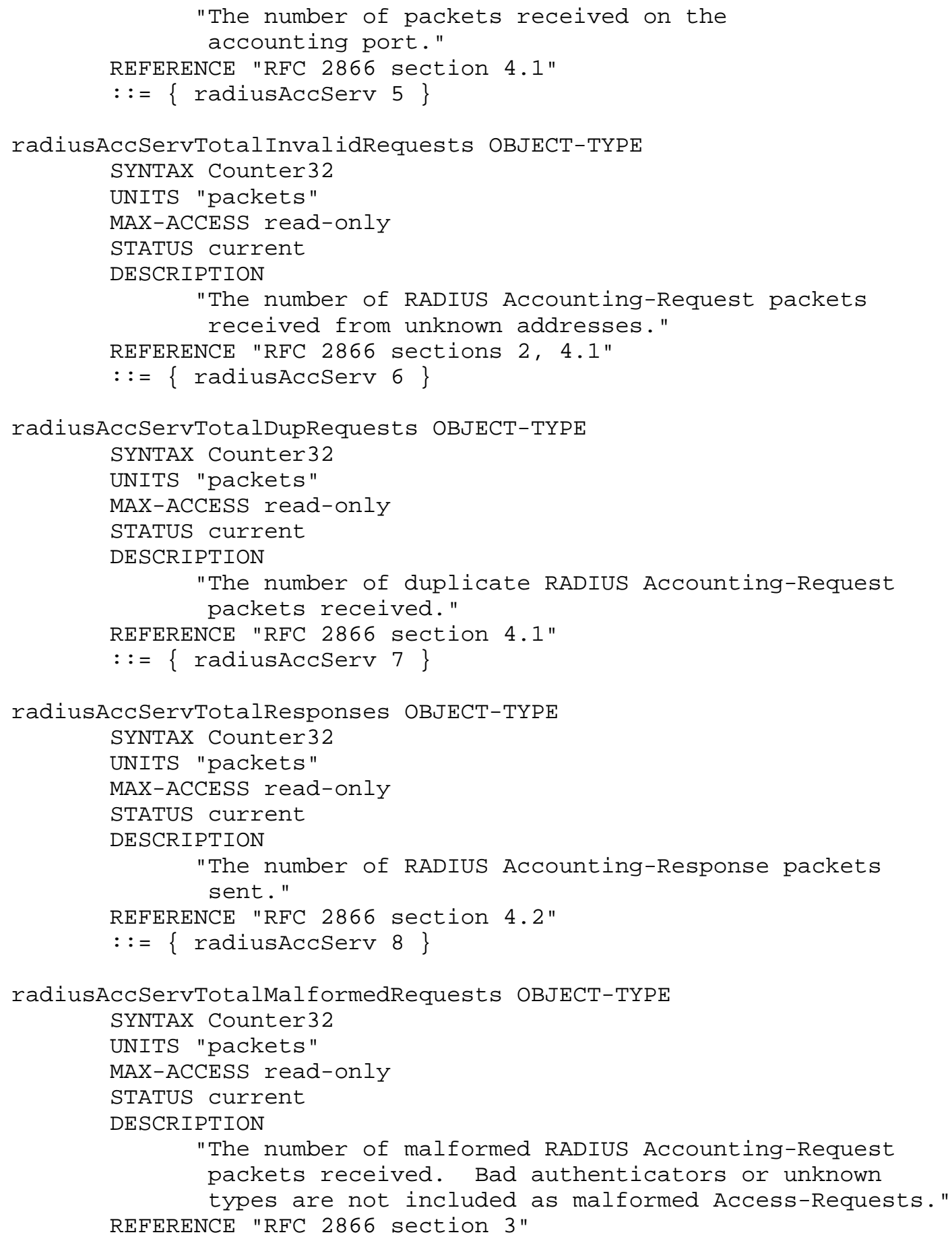




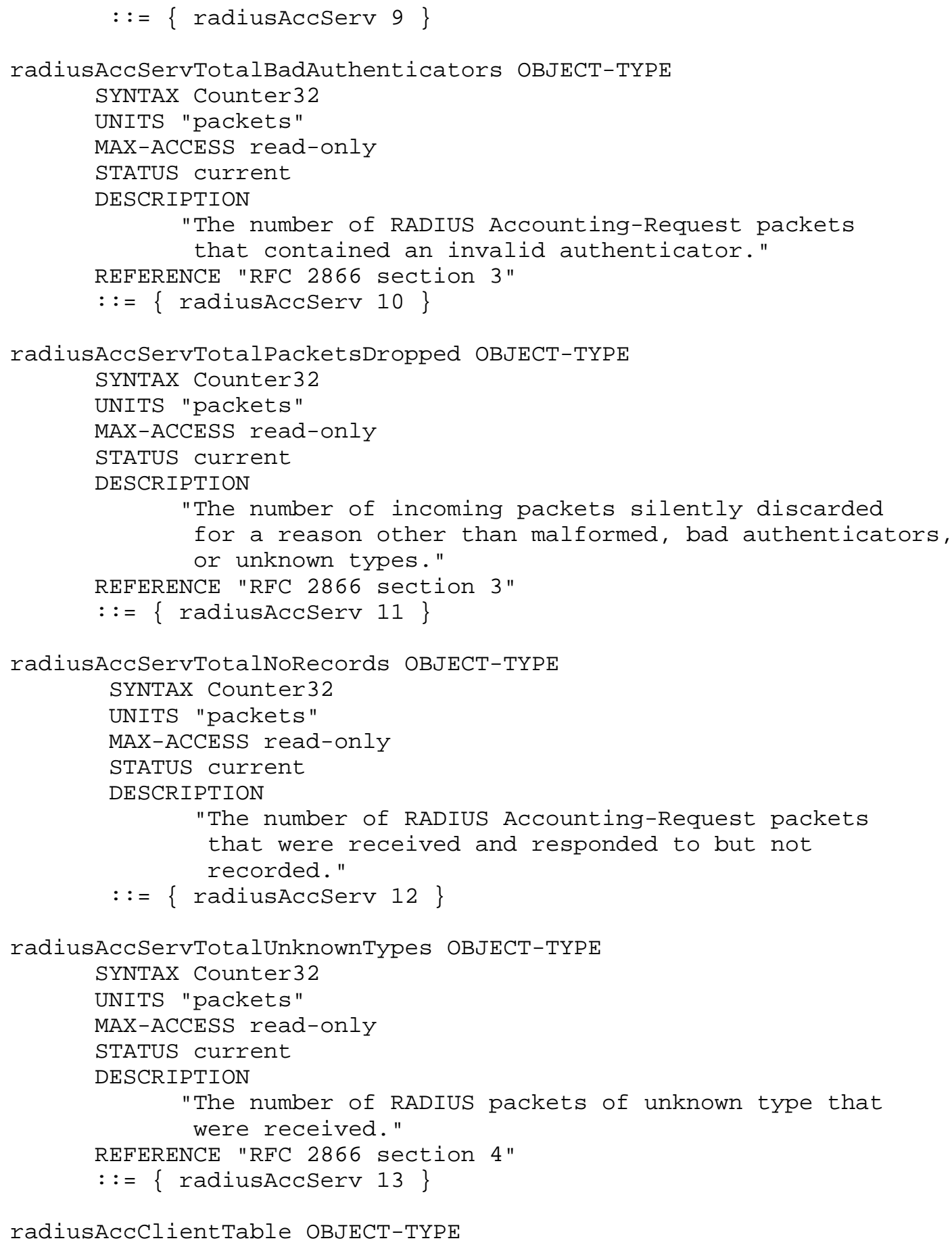




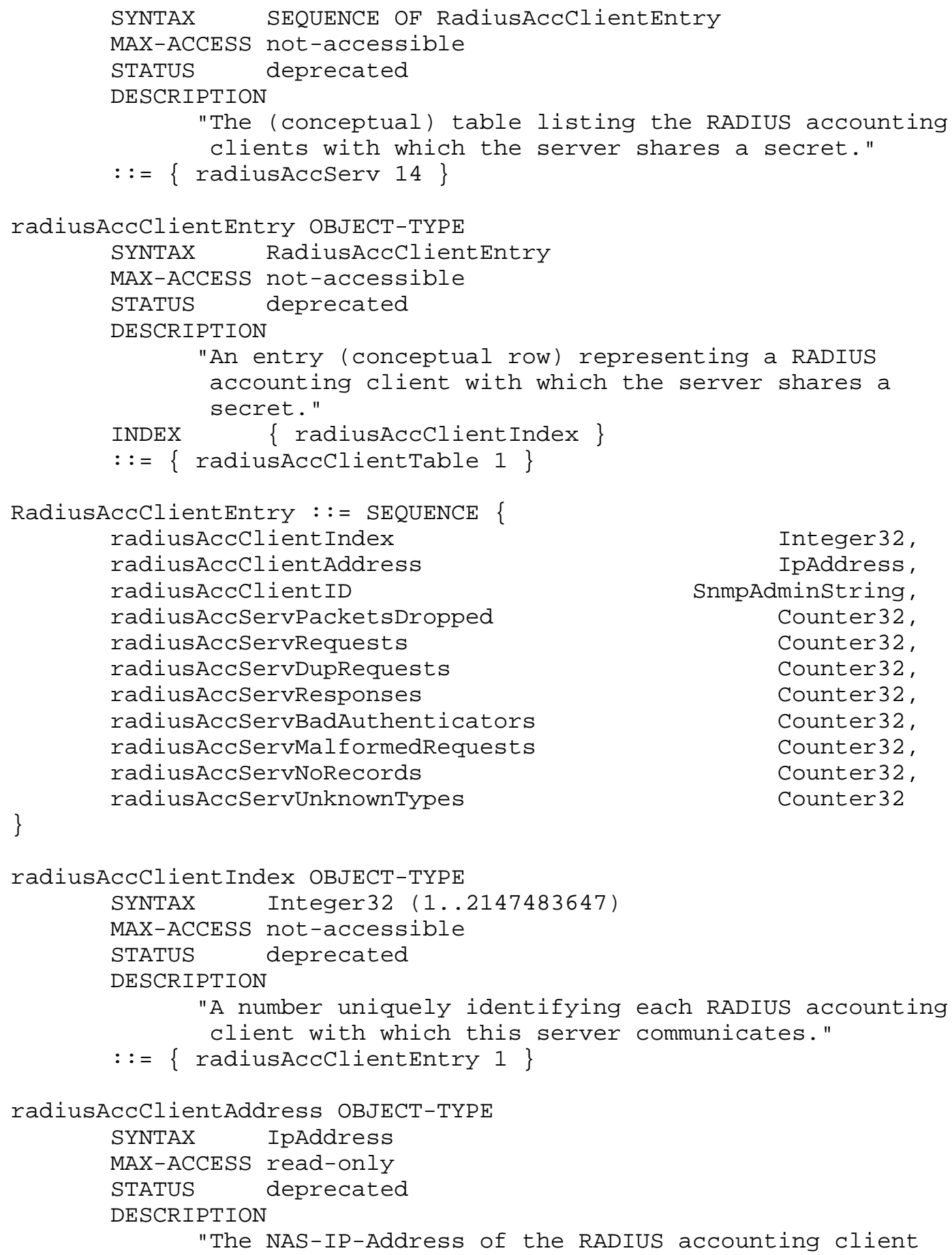




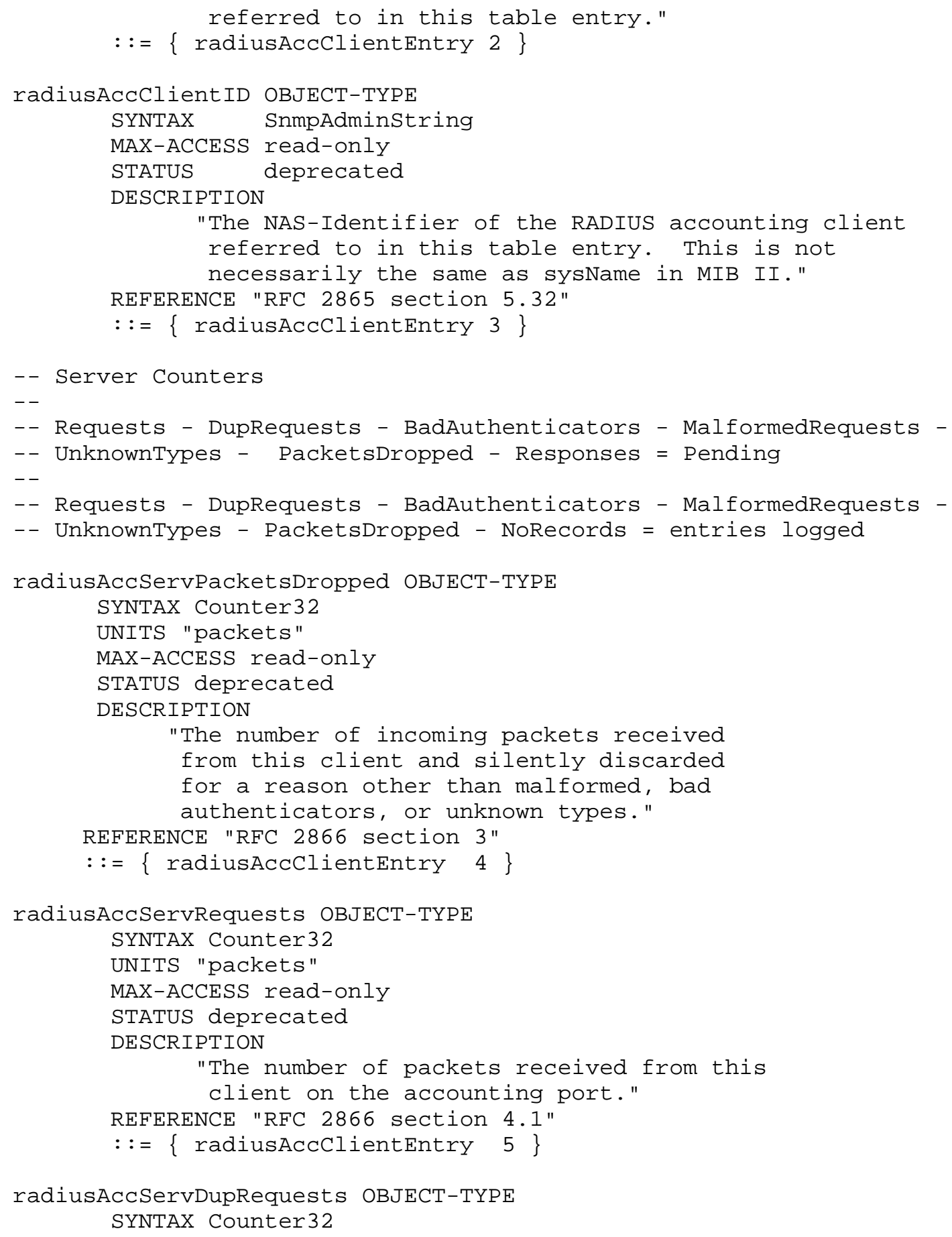




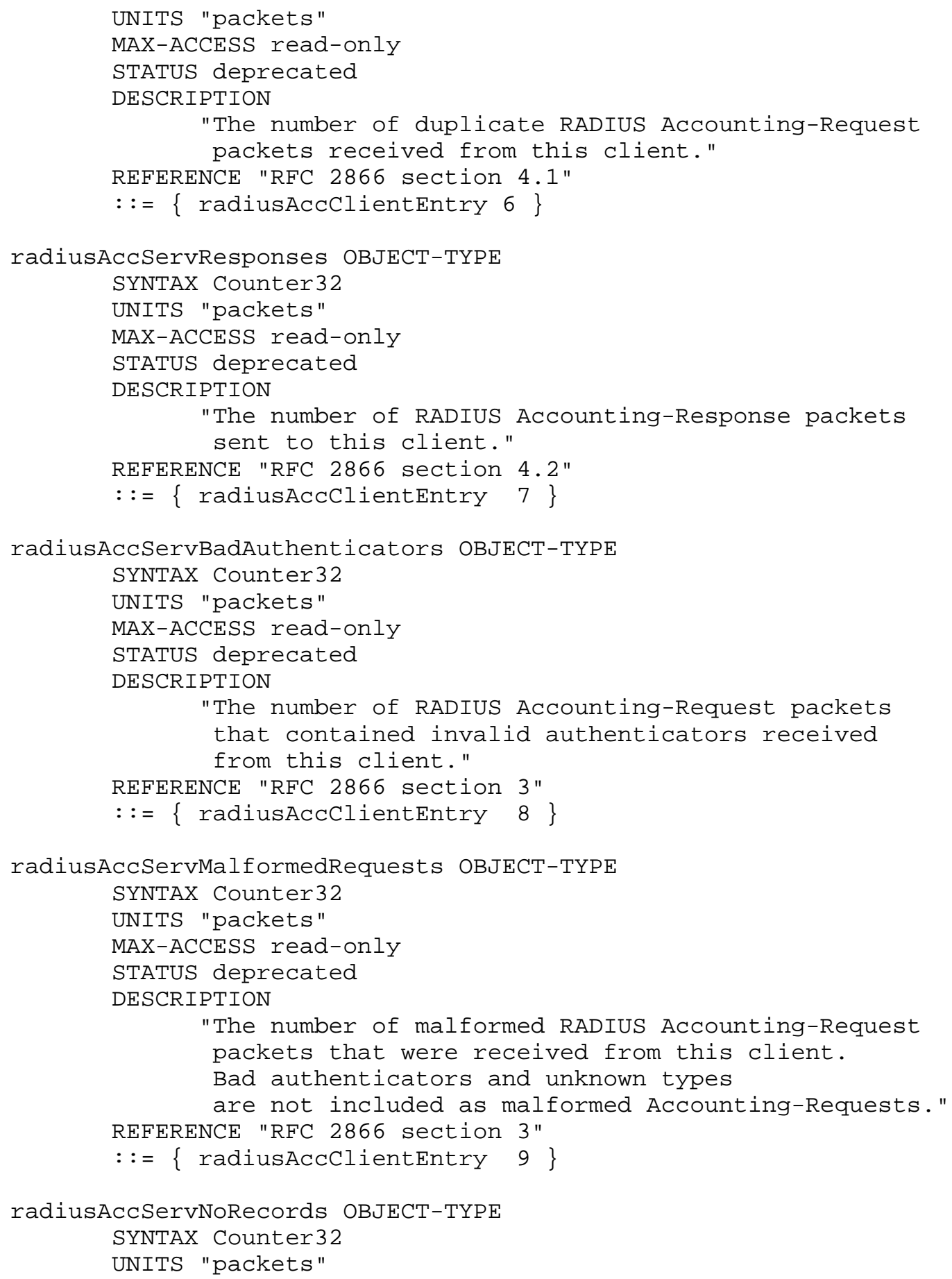




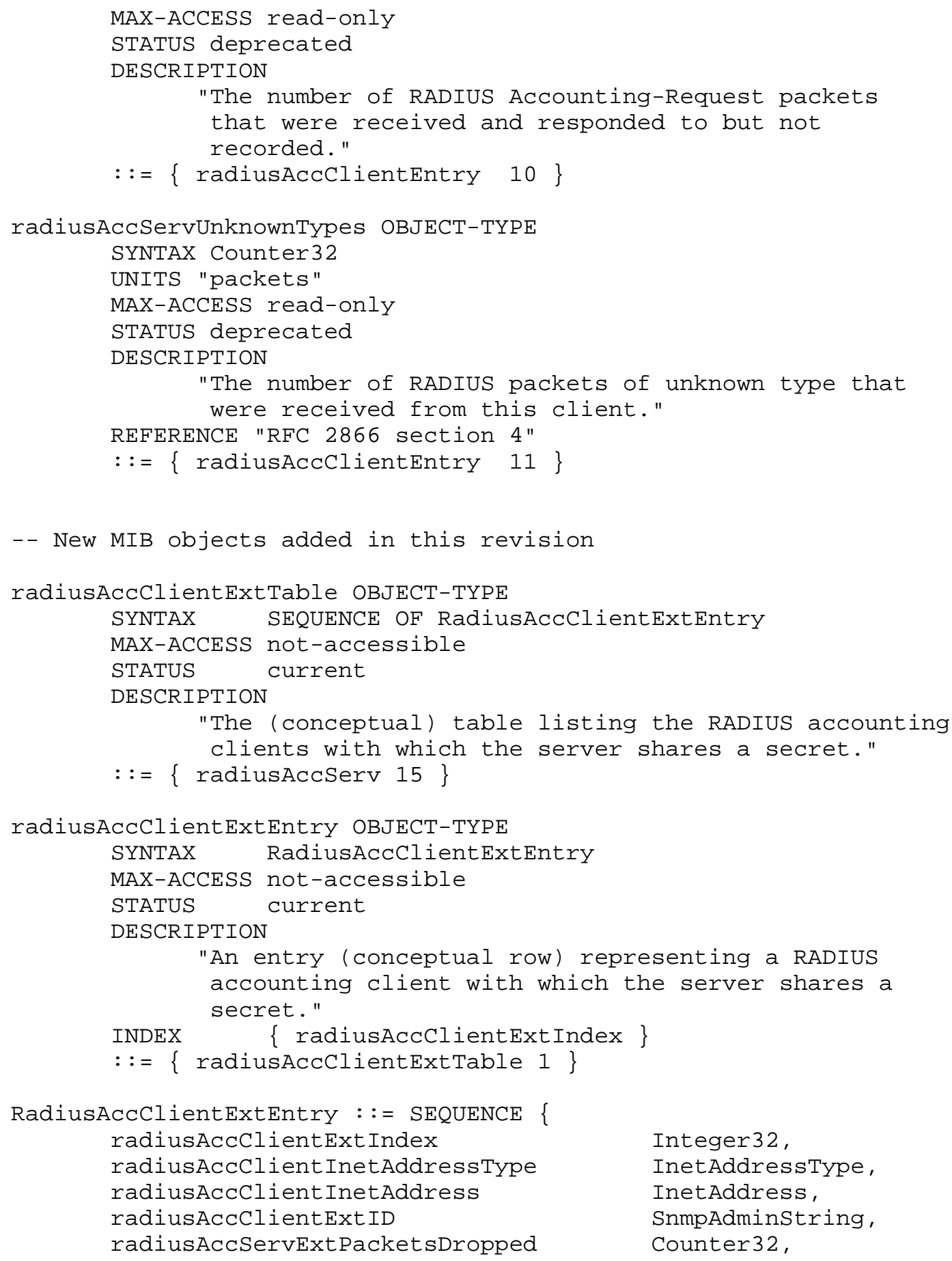




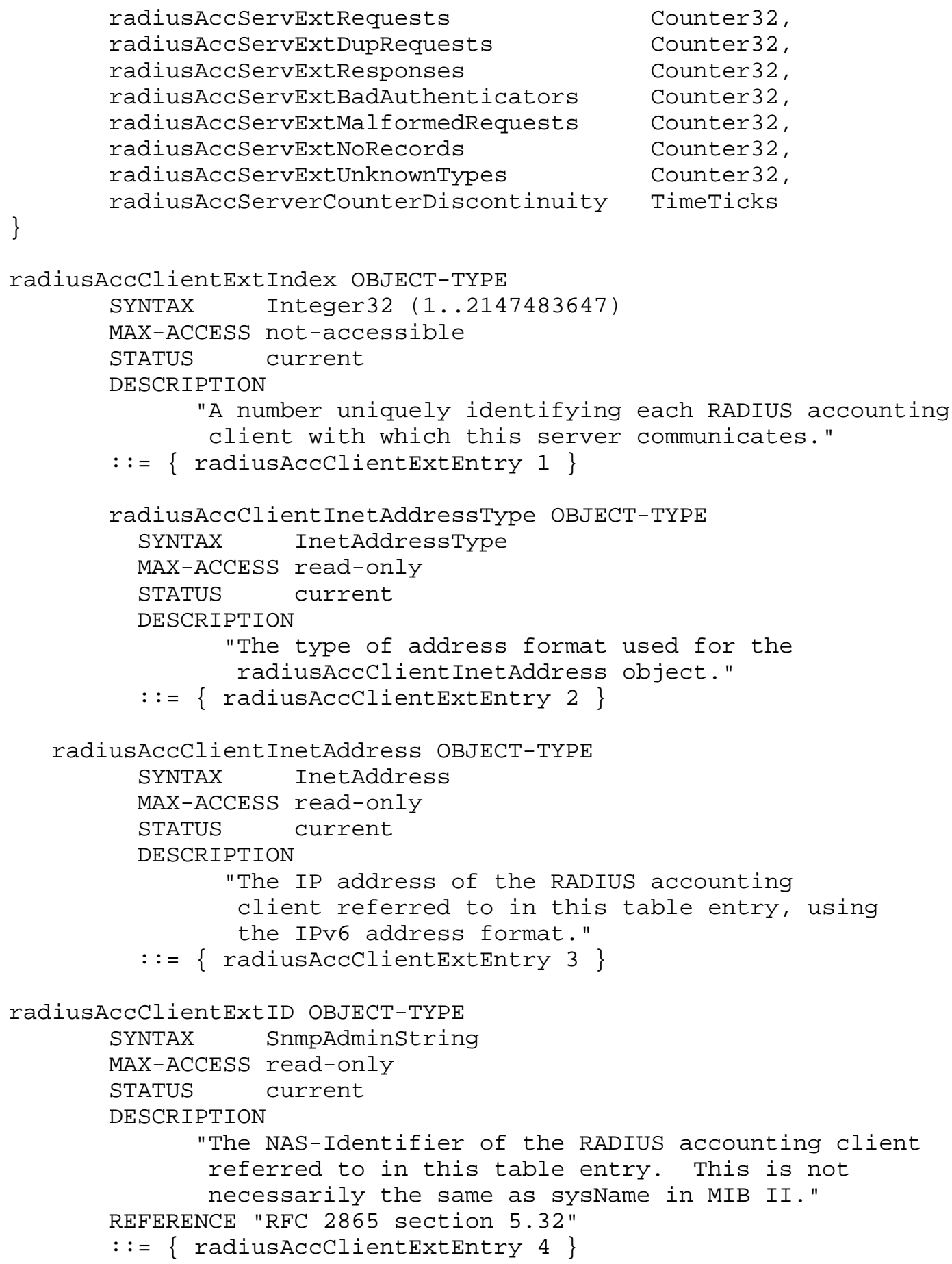

Nelson 


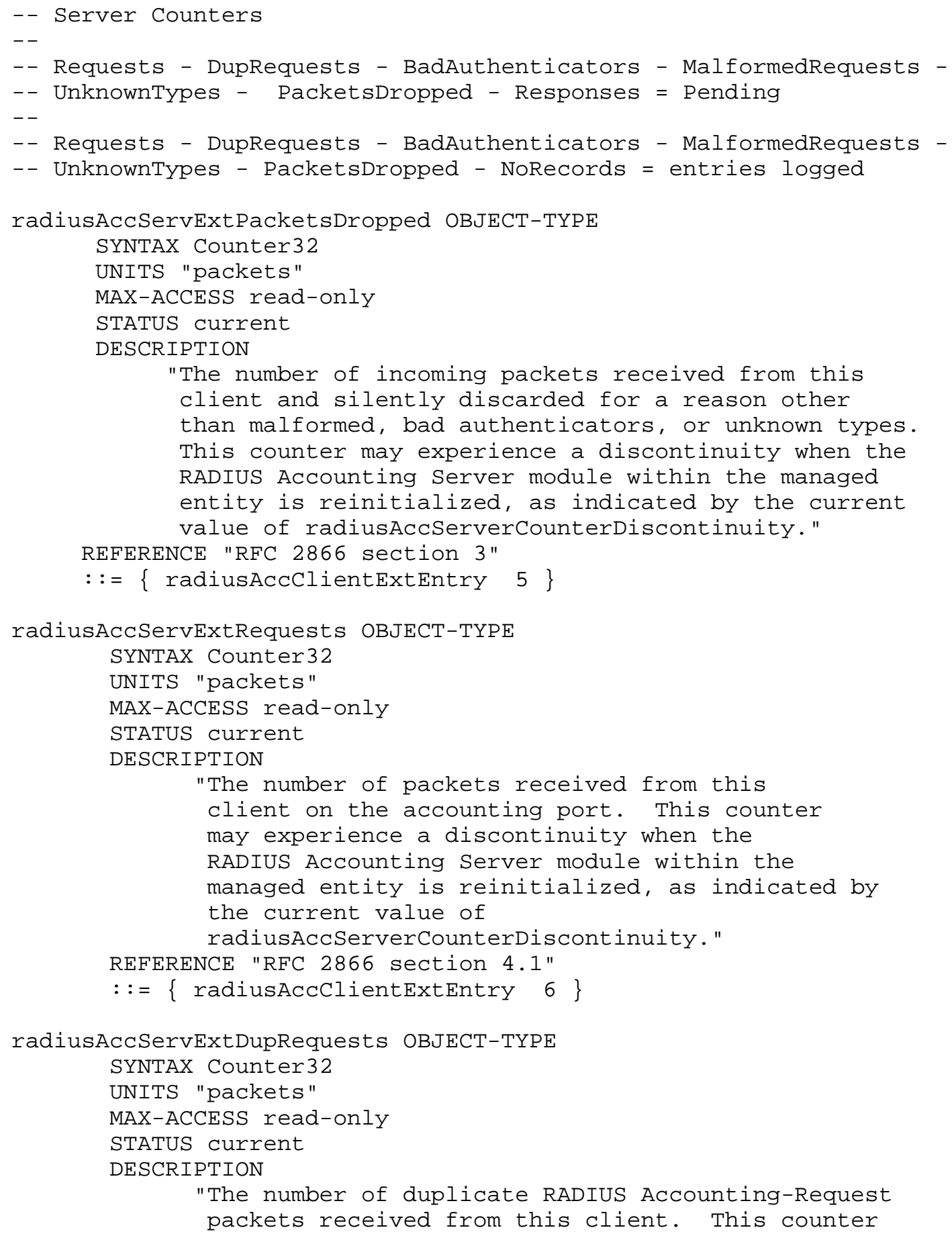




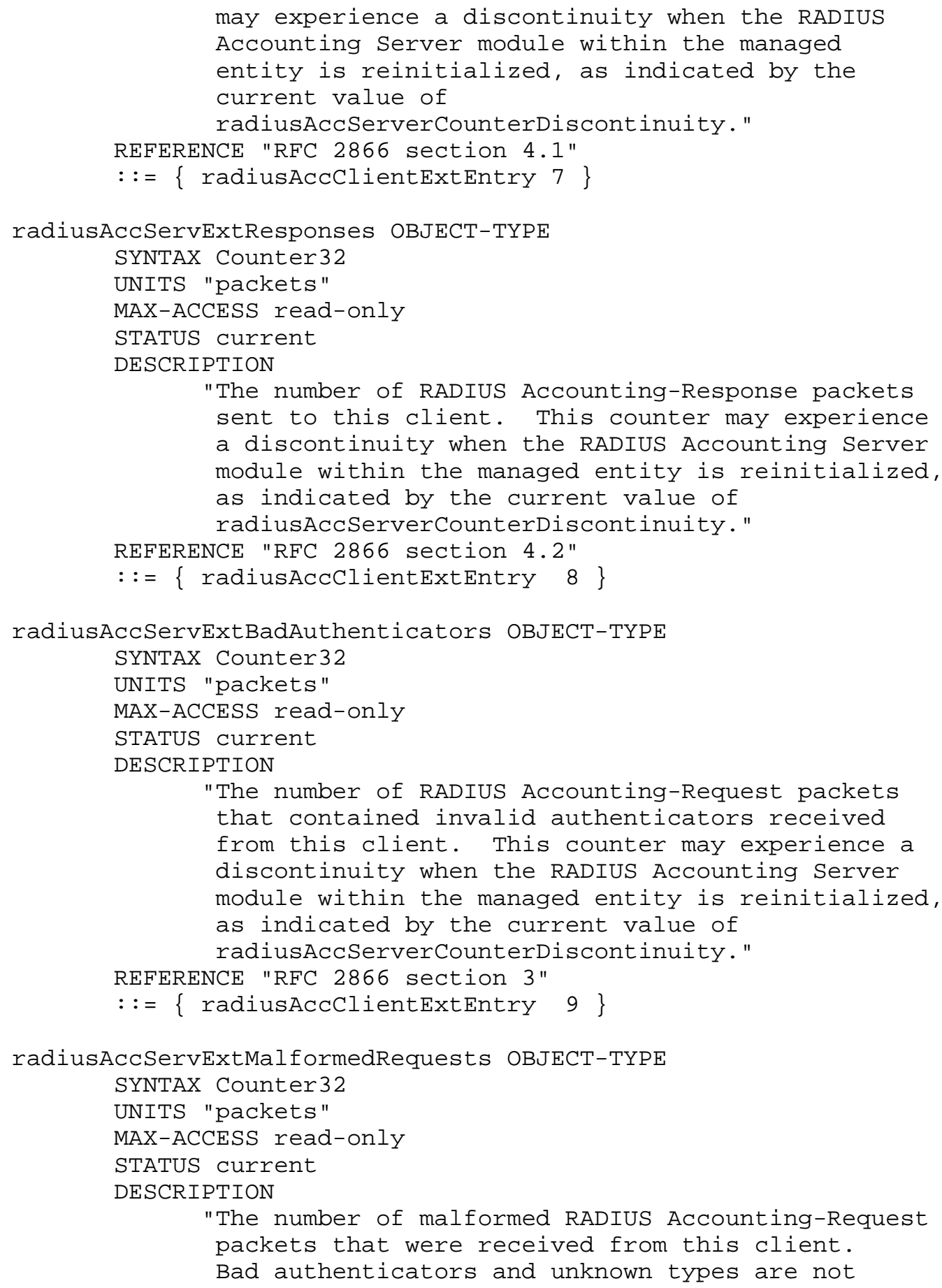

Nelson 


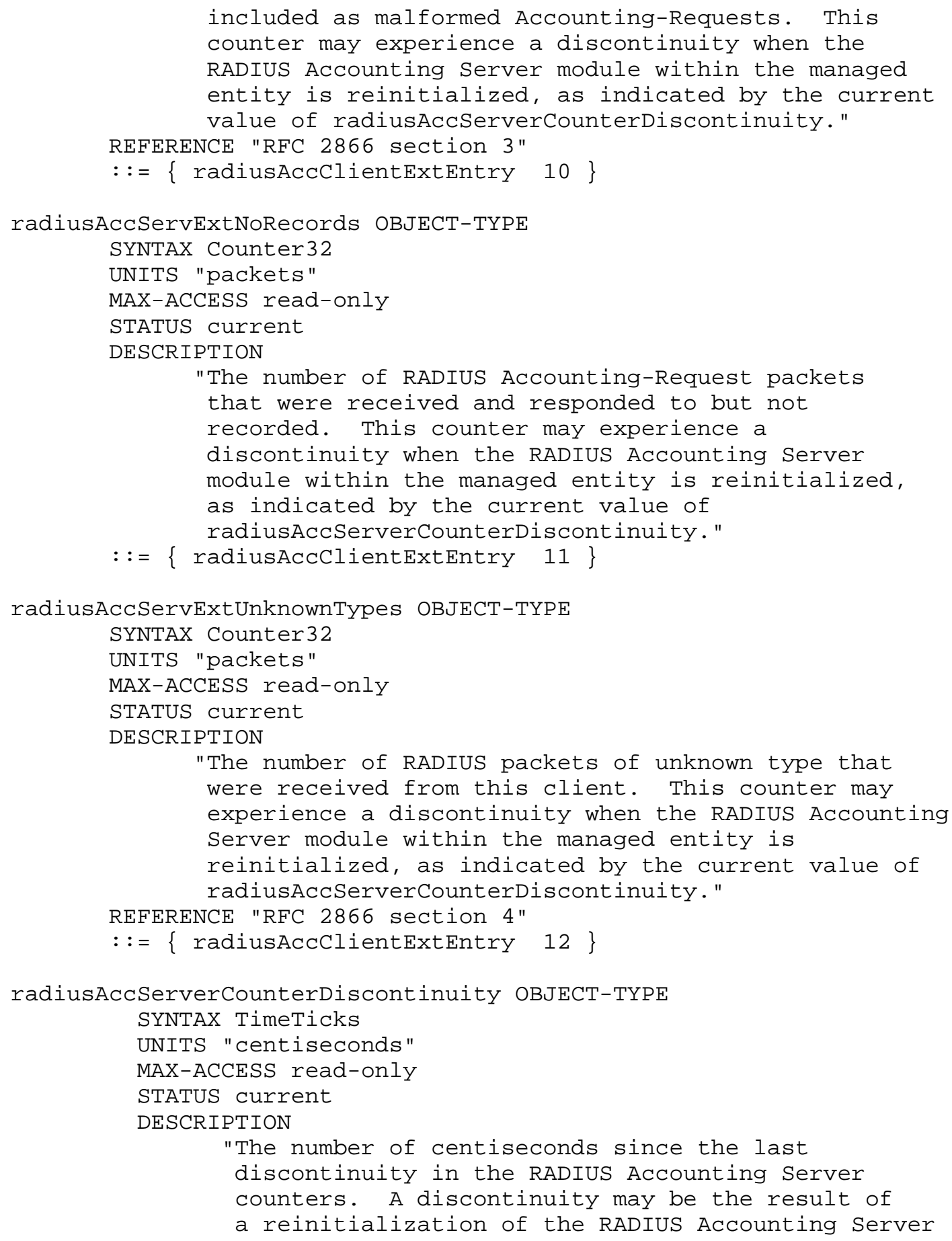




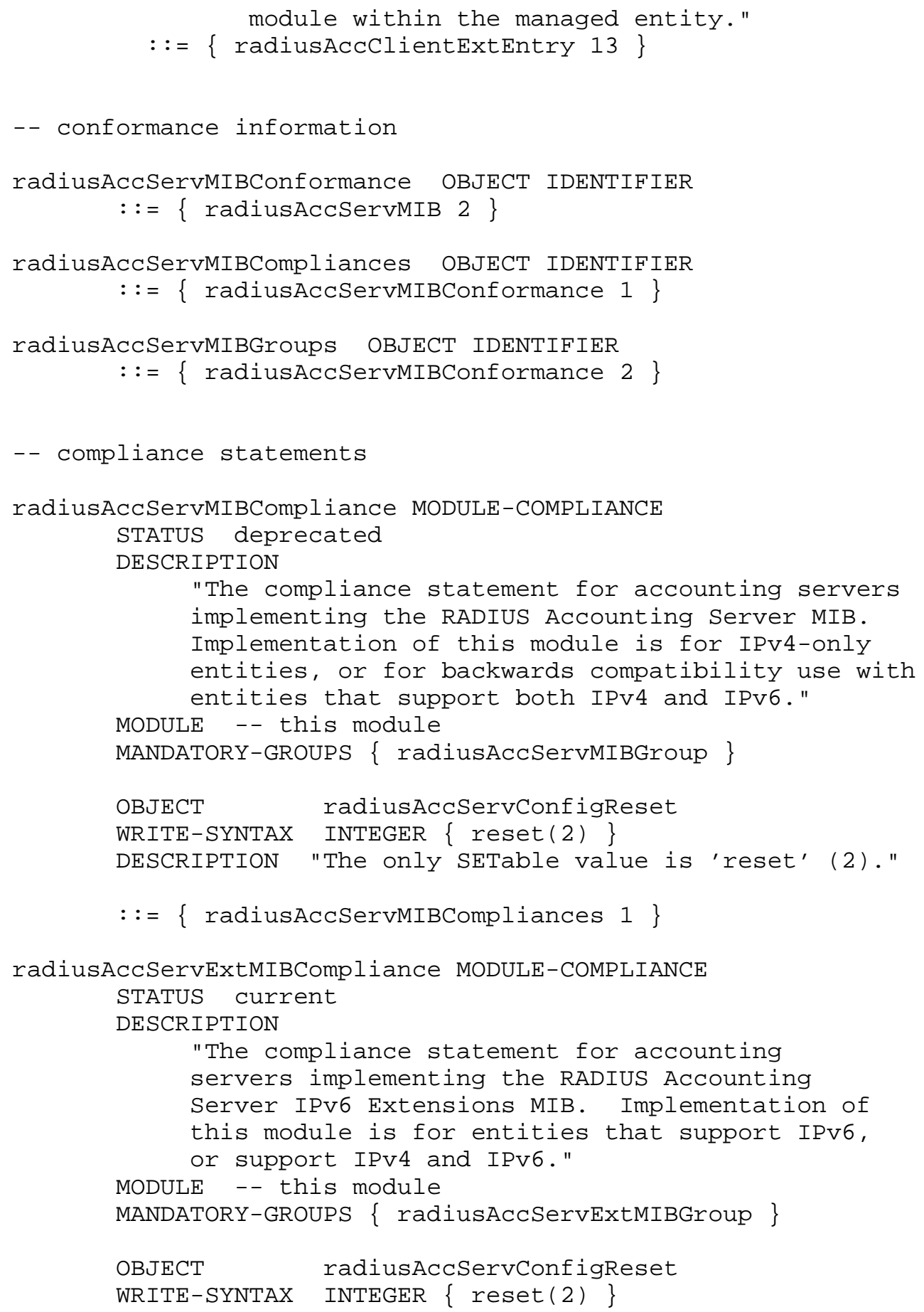




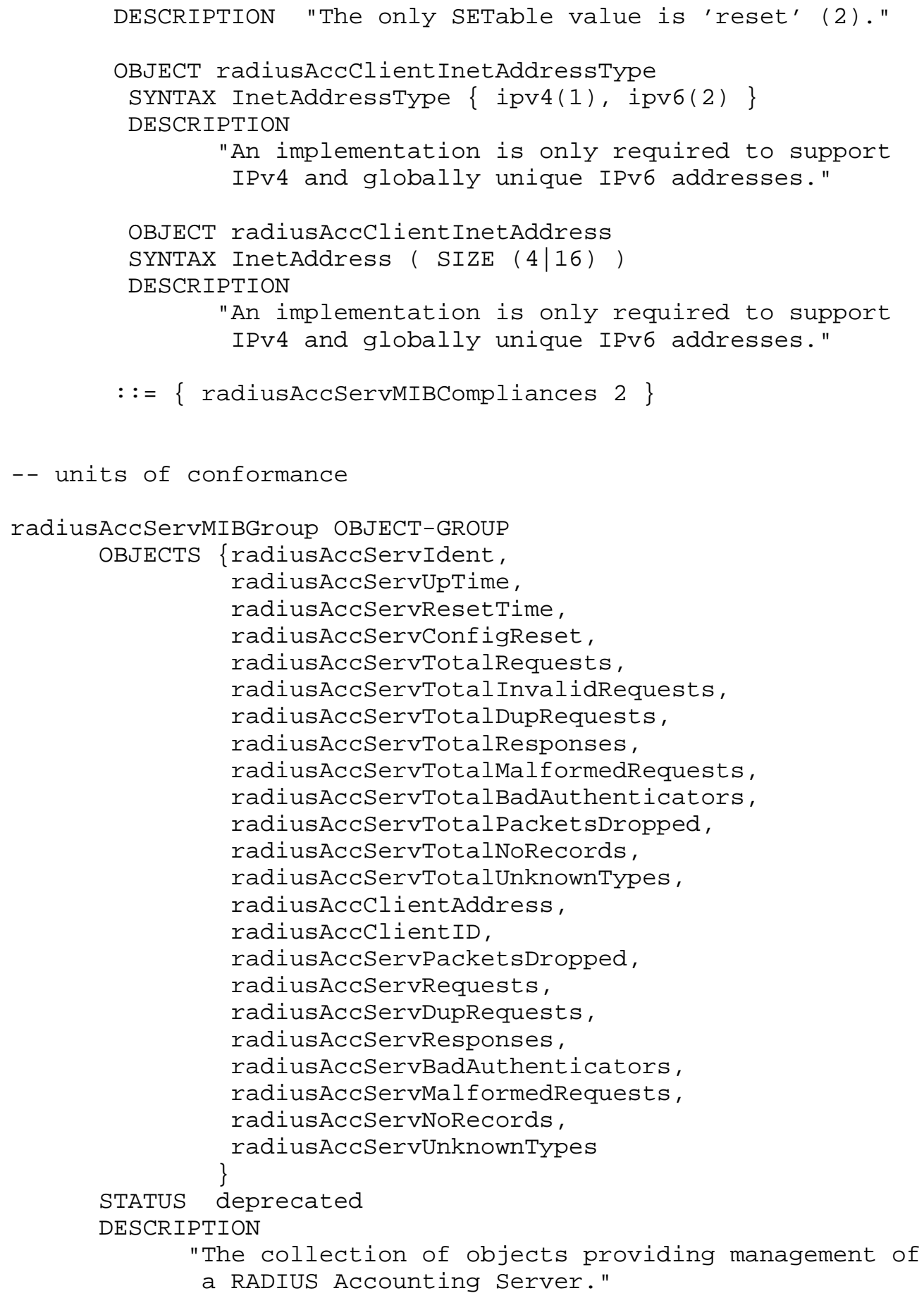




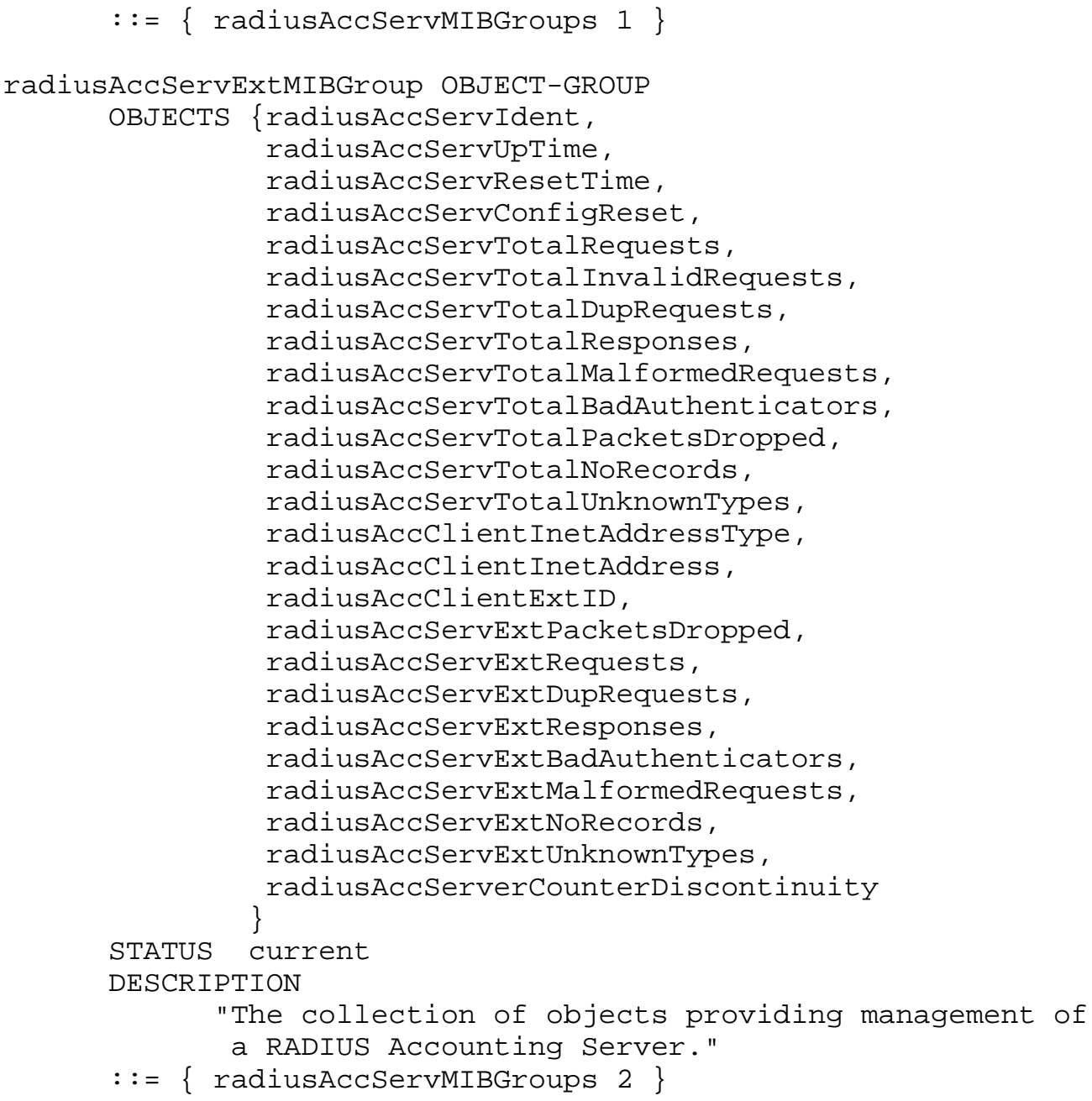

END

8. Security Considerations

There are management objects (radiusAccServConfigReset) defined in this MIB that have a MAX-ACCESS clause of read-write and/or readcreate. Such objects may be considered sensitive or vulnerable in some network environments. The support for SET operations in a nonsecure environment without proper protection can have a negative effect on network operations. These are: 


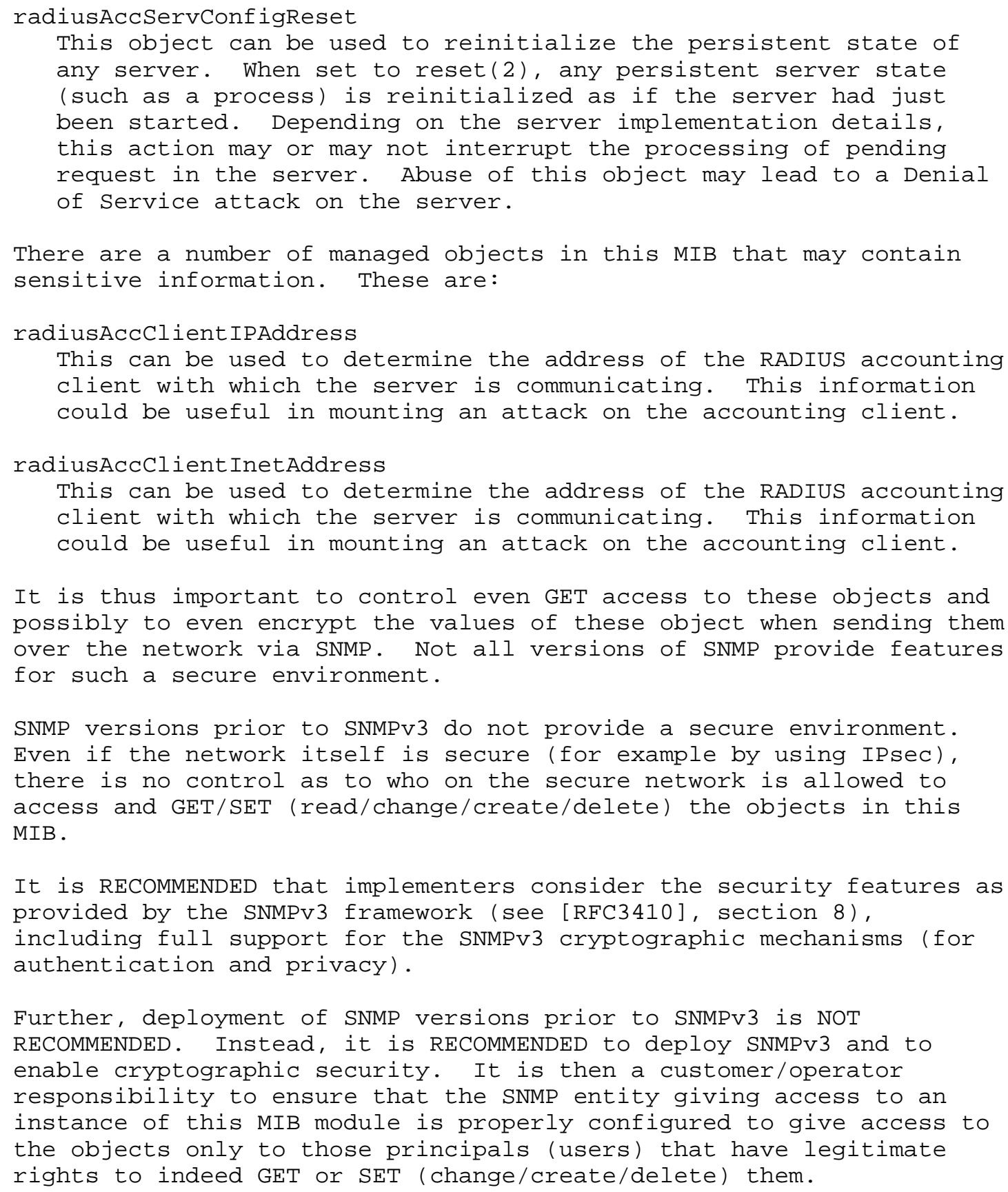




\section{References}

\subsection{Normative References}

[RFC2119] Bradner, S., "Key words for use in RFCs to Indicate Requirement Levels", BCP 14, RFC 2119, March 1997.

[RFC2578] McCloghrie, K., Ed., Perkins, D., Ed., and J. Schoenwaelder, Ed., "Structure of Management Information Version 2 (SMIv2)", STD 58, RFC 2578, April 1999.

[RFC2579] McCloghrie, K., Ed., Perkins, D., Ed., and J. Schoenwaelder, Ed., "Textual Conventions for SMIv2", STD 58, RFC 2579, April 1999.

[RFC2580] MCCloghrie, K., Perkins, D., and J. Schoenwaelder, "Conformance Statements for SMIv2", STD 58, RFC 2580, April 1999.

[RFC2866] Rigney, C., "RADIUS Accounting", RFC 2866, June 2000.

[RFC3411] Harrington, D., Presuhn, R., and B. Wijnen, "An Architecture for Describing Simple Network Management Protocol (SNMP) Management Frameworks", STD 62, RFC 3411, December 2002 .

[RFC4001] Daniele, M., Haberman, B., Routhier, S., and J. Schoenwaelder, "Textual Conventions for Internet Network Addresses", RFC 4001, February 2005.

9.2. Informative References

[RFC2621] Zorn, G. and B. Aboba, "RADIUS Accounting Server MIB", RFC 2621, June 1999.

[RFC2865] Rigney, C., Willens, S., Rubens, A., and W. Simpson, "Remote Authentication Dial In User Service (RADIUS)", RFC 2865, June 2000.

[RFC3410] Case, J., Mundy, R., Partain, D., and B. Stewart, "Introduction and Applicability Statements for InternetStandard Management Framework", RFC 3410, December 2002.

[RFC4670] Nelson, D., "RADIUS Accounting Client MIB for IPv6", RFC 4670, August 2006 . 
Appendix A. Acknowledgements

The authors of the original MIB are Bernard Aboba and Glen Zorn.

Many thanks to all reviewers, especially to Dave Harrington, Dan

Romascanu, C.M. Heard, Bruno Pape, Greg Weber, and Bert Wijnen.

Author's Address

David B. Nelson

Enterasys Networks

50 Minuteman Road

Andover, MA 01810

USA

EMail: dnelson@enterasys.com 
Full Copyright statement

Copyright (C) The Internet Society (2006).

This document is subject to the rights, licenses and restrictions contained in BCP 78, and except as set forth therein, the authors retain all their rights.

This document and the information contained herein are provided on an "AS IS" basis and THE CONTRIBUTOR, THE ORGANIZATION HE/SHE REPRESENTS OR IS SPONSORED BY (IF ANY), THE INTERNET SOCIETY AND THE INTERNET ENGINEERING TASK FORCE DISCLAIM ALL WARRANTIES, EXPRESS OR IMPLIED, INCLUDING BUT NOT LIMITED TO ANY WARRANTY THAT THE USE OF THE INFORMATION HEREIN WILL NOT INFRINGE ANY RIGHTS OR ANY IMPLIED WARRANTIES OF MERCHANTABILITY OR FITNESS FOR A PARTICULAR PURPOSE.

Intellectual Property

The IETF takes no position regarding the validity or scope of any Intellectual Property Rights or other rights that might be claimed to pertain to the implementation or use of the technology described in this document or the extent to which any license under such rights might or might not be available; nor does it represent that it has made any independent effort to identify any such rights. Information on the procedures with respect to rights in RFC documents can be found in BCP 78 and BCP 79 .

Copies of IPR disclosures made to the IETF Secretariat and any assurances of licenses to be made available, or the result of an attempt made to obtain a general license or permission for the use of such proprietary rights by implementers or users of this specification can be obtained from the IETF on-line IPR repository at http://www.ietf.org/ipr.

The IETF invites any interested party to bring to its attention any copyrights, patents or patent applications, or other proprietary rights that may cover technology that may be required to implement this standard. Please address the information to the IETF at ietf-ipreietf.org.

Acknowledgement

Funding for the RFC Editor function is provided by the IETF Administrative Support Activity (IASA). 